\title{
Vérification expérimentale de la formule de Gassmann dans les calcaires poreux
}

\author{
P. Rasolofosa on ${ }^{1}$ et B. Zinszner ${ }^{1}$ \\ 1 Institut français du pétrole, 1 et 4, avenue de Bois-Préau, 92852 Rueil-M almaison Cedex - France \\ e-mail : patrick.rasolofosaon@ifp.fr - bernard.zinszner@ifp.fr
}

\begin{abstract}
Résumé - Dans cet article, nous présentons une vérification expérimentale, originale et convaincante, de la formule de Gassmann. Cette dernière permet de calculer l'effet du fluide saturant l'espace poreux d'une roche, sur les vitesses sismiques. La méthode utilisée pour cette vérification consiste en la mesure précise de la variation de vitesse de propagation des ondes ultrasonores $P$ et $S$, lors de la substitution de liquides de module d'incompressibilité variable, dans six roches calcaires et dans une lave perméable. On peut ainsi montrer l'existence d'une relation expérimentale linéaire entre le module d'incompressibilité de la roche $\left(K_{\text {sat }}\right)$ et le module d'incompressibilité du liquide saturant $\left(K_{\mathrm{fl}}\right)$. Nous montrons que cette relation linéaire constitue une simplification de la formule de Gassmann tout à fait acceptable, quantitativement, pour les roches poreuses. L'ordonnée à l'origine de cette relation linéaire est égale au module d'incompressibilité de la roche sèche $\left(K_{\text {dry }}\right)$ et sa pente est directement liée au coefficient $\beta$ de Biot. On dispose donc d'un moyen de contrôle quantitatif par l'estimation du module d'incompressibilité du minéral formant la roche $\left(K_{\text {grain }}\right)$. Dans le cas de la calcite, constituant les échantillons de calcaire, la valeur de ce paramètre est bien connue. Sur un échantillon de calcaire, nous avons aussi pratiqué des substitutions de fluide en système diphasique (eau plus liquide non miscible à l'eau). Dans ce cas, la formule est encore vérifiée lorsque l'on prend pour valeur de la compressibilité du fluide $\left(1 / K_{\mathrm{fl}}\right)$, la moyenne des compressibilités des fluides présents, pondérées par leur fraction volumique.
\end{abstract}

Abstract - Experimental Verification of Gassmann's Equation in Porous Limestone - In this paper, an original and convincing experimental verification of Gassmann's Equation is presented. Gassmann's Equation allows the computation of the impact of saturating fluid substitution on seismic velocity in rocks. The experimental verification is based on the accurate measurement of $P$ and $S$ ultrasonic wave velocities when substituting in the pores of the rock, several liquids of different bulk modulus. The measurements were performed on six limestone and one porous lava samples. The experimental data show a linear relationship between the saturated rock bulk modulus $\left(K_{\text {sat }}\right)$ and the fluid bulk modulus $\left(K_{f l}\right)$. We show that this linear relationship results from a reasonable simplification of Gassmann's Equation. The limit value of $K_{\text {sat }}$ for vanishing $K_{f l}$ is obviously $K_{d r y}$ and the slope of the curve $K_{\text {sat }}$ vs $K_{f l}$ is a function of Biot's coefficient $\beta$. Thus we have a simple way to check the experimental result by comparing the value of the bulk modulus of the rock forming grain $\left(K_{\text {grain }}\right)$ derived from $\beta$ and the known value for well identified mineral as calcite, in our experiments. In one sample, we performed a diphasic fluid substitution, using water plus non water-miscible fluids. In this case, the Gassmann's Equation is also verified when taking for fluid compressibility $\left(1 / K_{f l}\right)$, the fluids compressibility average, weighted by their respective volume fraction. 


\section{IN TRO DUCTIO N}

La formule de Gassmann, très utile en géophysique pour prévoir l'effet du fluide saturant l'espace poreux d'une roche sur les vitesses sismiques, bénéficie d'un regain d'intérêt avec le développement de la sismique répétitive (ou monitoring sismique, ou sismique 4D). Cette sismique a pour but de détecter, dans les gisements pétroliers ou gaziers en production, les variations dans la nature des fluides présents dans le réservoir.

La formule de Gassmann a fait l'objet de très nombreux essais expérimentaux, le plus souvent fondés sur la comparaison des vitesses de propagation des ondes ultrasonores dans la roche à l'état sec et à l'état saturé d'eau. Des essais plus poussés reposent sur la mesure les vitesses de propagation pour divers états de saturation en gaz. On peut trouver dans Cadoret et al. (1995), un exemple de ce type. Ces expériences ont ceci de frustrant, que la différence entre l'état avec ou sans gaz, est si importante que la comparaison des résultats quantitatifs peut s'accommoder de grandes incertitudes.

Nous présentons ici une approche différente, fondée sur la comparaison des modules d'incompressibilité de la roche $\left(K_{\text {sat }}\right)$, pour des états de saturation en différents liquides de modules d'incompressibilités $\left(K_{\mathrm{fl}}\right)$ différents. Dans ce cas, les différences entre les $K_{\mathrm{fl}}$ sont assez faibles mais progressives. On peut donc comparer beaucoup plus finement l'évolution de la relation $K_{\text {sat }}$ fonction de $K_{\mathrm{fl}}$, qui constitue le point central de notre vérification.

La vérification au travers de la relation $K_{\text {sat }}=f\left(K_{\mathrm{fl}}\right)$ est grandement facilitée par le fait que la formule de Gassmann peut se simplifier en une relation linéaire de type $K_{\mathrm{sat}}=A K_{\mathrm{fl}}$ $+K_{\text {dry }}, K_{\text {dry }}$ étant le module d'incompressibilité de la roche saturée d'un fluide très compressible (gaz). Nous montrons que cette simplification est tout à fait acceptable pour les roches poreuses (et particulièrement les calcaires).

En appliquant cette méthode à six échantillons de calcaire (et un échantillon de lave poreuse), nous pensons apporter une preuve expérimentale convaincante de la validité de la formule de Gassmann.

\section{LA FORMULE DE GASSMAN N}

\subsection{Présentation résumée du cas général}

La formule de Gassmann, très utile en géophysique pour prévoir l'effet, sur les vitesses sismiques, d'une variation de fluide saturant, a fait l'objet de très nombreuses publications. Nous renverrons le lecteur intéressé par une étude détaillée à Bourbié et al. (1986) ou Mavko et al. (1998).

Nous nous limiterons ici à présenter quelques points utiles à la compréhension de la suite.

Gassmann (1951) a calculé, pour une sollicitation quasi statique, la différence de module entre un milieu poreux drainé (c'est-à-dire dans lequel la pression du fluide saturant reste indépendante de la sollicitation) et non drainé, dans lequel la variation de pression du fluide saturant reprend une partie de la sollicitation mécanique. La théorie de Gassmann suppose un milieu poreux continu et homogène, mais n'implique aucune condition sur la géométrie des pores.

L'application de la formule de Gassmann à la propagation d'onde (cas dynamique) demande que la fréquence soit suffisamment basse pour que la variation de pression de fluide saturant induite par le passage de l'onde soit homogène dans le milieu poreux (absence de gradient).

La formule de Gassmann relie les modules d'incompressibilité drainé $\left(K_{\text {dry }}\right)$ et non drainé $\left(K_{\text {sat }}\right)$, à l'aide de paramètres propres :

- au milieu poreux : porosité $\phi$ et incompressibilité du minéral formant les grains $\left(K_{\text {grain }}\right)$;

- au fluide saturant: $K_{\mathrm{fl}}$, module d'incompressibilité du fluide.

Le module d'incompressibilité drainé $\left(K_{\text {dry }}\right)$, est associé à celui de la roche saturée de gaz sous faible pression (roche sèche). Il est important de noter qu'expérimentalement, le module mesuré sur une roche sèche peut être très différent du module $K_{\text {dry }}$ dont traite Gassmann. En effet, en atmosphère terrestre, une roche « sèche » contient toujours de l'eau adsorbée qui peut avoir une influence considérable sur les forces capillaires aux joints de grains. Les déboires des expérimentateurs qui mesurèrent les paramètres acoustiques des premières roches lunaires sont une illustration de ce phénomène (voir par exemple Tittmann et al., 1980). Il est plus prudent de considérer que $K_{\text {dry }}$ correspond au module mesuré sur une roche contenant une saturation en eau suffisante pour limiter considérablement la pression capillaire (saturation en eau de plusieurs pour-cent).

Une expression pratique de la formule de Gassmann fait intervenir le coefficient $\beta$ de Biot (1956) :

avec :

$$
K_{\mathrm{sat}}=K_{\mathrm{dry}}+\beta^{2} M
$$

$$
\beta=1-\left(K_{\text {dry }} / K_{\text {grain }}\right)
$$

et :

$$
M^{-1}=\left[(\beta-\phi) / K_{\text {grain }}\right]+\phi / K_{\mathrm{fl}}
$$

Rappelons que, les fluides ne reprenant pas les cisaillements, les modules de cisaillement drainé $\left(\mu_{\text {dry }}\right)$ ou non drainé $\left(\mu_{\text {sat }}\right)$ sont égaux.

\subsection{Linéarisation de la formule de Gassmann (roches poreuses à forte incompressibilité de grain)}

Dans l'expression précédente, on peut noter que le premier terme de l'expression de $M^{-1}$ est souvent nettement plus petit que le second puisque $K_{\text {grain }}$ est toujours beaucoup plus grand que $K_{\mathrm{fl}}$. En négligeant ce premier terme, on obtient une expression très simple :

$$
K_{\mathrm{sat}} \approx K_{\mathrm{dry}}+\left(\beta^{2} / \phi\right) K_{\mathrm{fl}}
$$


On peut estimer l'erreur ainsi commise en calculant l'écart entre les deux expressions pour des valeurs correspondant à des paramètres $K_{\text {dry }}$ et $K_{\text {grain }}$ typiques de calcaires et de grès. Les résultats (fig. 1 ) montrent que pour les calcaires (matrice en calcite) de porosité supérieure ou égale à $20 \%$, les erreurs induites par la formule simplifiée (4) sont bien inférieures à $5 \%$ en termes de module élastique, et bien inférieures à $2,5 \%$ en termes de vitesse pour des fluides usuels (incompressibilité inférieure à $3 \mathrm{GPa}$ ). Ces erreurs sont négligeables par rapport aux incertitudes expérimentales.

\section{CONDITIONS POUR UNE VÉRIFICATION EXPÉRIMENTALE}

La formule simplifiée (4) permet d'envisager une vérification expérimentale simple de la formule de Gassmann. En effet, en substituant des fluides d'incompressibilité variée dans un milieu poreux dont on mesurerait les vitesses de propagation des ondes de compression ( $V p)$ et de cisaillement $(V s)$, il serait facile de tracer la relation expérimentale $K_{\text {sat }}$ en fonction de $K_{\mathrm{fl}}$. La linéarité de cette relation serait un premier élément de preuve à associer à la vérification de la valeur de $\beta^{2} / \phi$ (pente de la droite) et $K_{\text {dry }}$ expérimental (ordonnée à l'origine).

Rappelons que $\mu=\rho V s^{2}$ et que $K=\rho V p^{2}-(4 / 3) \mu$, $\rho$ étant la masse volumique du milieu considéré.

Nous avons vu que l'expression simplifiée était d'autant plus satisfaisante que $K_{\text {grain }}$ et la porosité étaient élevés ; cette observation nous conduit tout naturellement à utiliser des calcaires poreux $\left(K_{\text {grain }}=70 \mathrm{GPa}\right.$, pour la calcite $)$.
Le choix des calcaires est motivé par plusieurs autres raisons importantes :

- les géométries poreuses des calcaires sont, à juste titre, connues pour leur complexité (microporosité ubiquiste) ;

- la calcite est peu réactive aux composés organiques divers que nous devrons utiliser pour obtenir des incompressibilités variables. Nous serons donc à l'abri des problèmes redoutables que pose la fraction argileuse des grès lorsqu'on la met en contact avec de l'eau douce, de l'éthanol, de l'éthylène glycol, etc. ;

- les vitesses acoustiques des calcaires sont généralement beaucoup moins sensibles que celles des grès à la pression de confinement (absence de joint de grain). Ceci nous permettra de travailler à la pression atmosphérique et évitera ainsi l'immobilisation longue de cellules de mesure lors des déplacements miscibles de liquides, déplacements nécessairement lents si l'on veut être sûr d'un résultat convenable dans des milieux poreux complexes.

\section{MÉTHODE EXPÉRIMENTALE}

\subsection{Roches utilisées}

Nous avons sélectionné six roches calcaires poreuses ayant fait l'objet de nombreuses études antérieures et donc bien connues. Le lecteur intéressé pourra trouver une description détaillée, notamment des courbes porosimétriques de grande qualité ainsi que de nombreuses caractérisations acoustiques
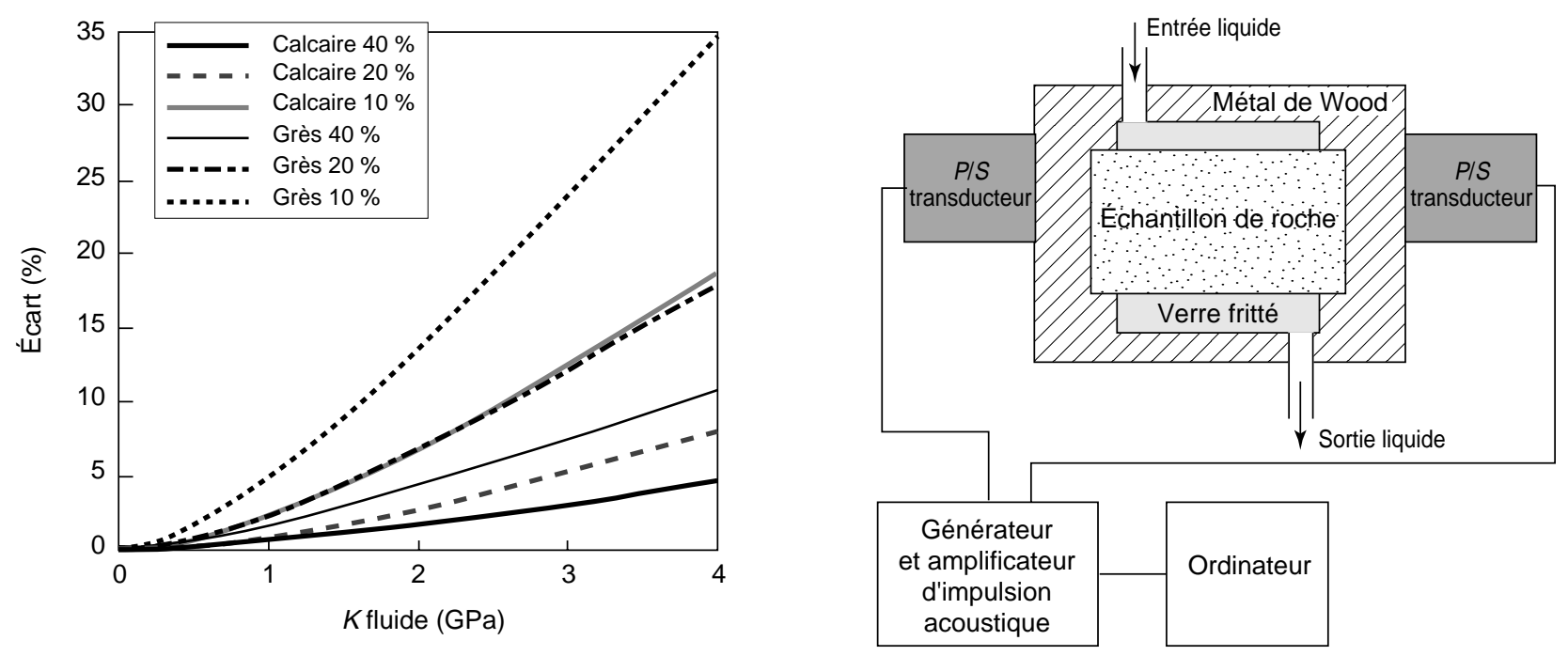

Figure 1

Comparaison des résultats de la formule de Gassmann complète et linéarisée.

Comparison of the results of complete and linearized Gassmann's Equation.

Figure 2

Schéma du dispositif expérimental. Sketch of the experimental set-up. 
dans les thèses de T. Cadoret (1993, porosimétrie) et $\mathrm{N}$. Lucet (1989). Nous avons aussi étudié par comparaison une lave poreuse présentant un réseau poreux très différent. Nous donnons dans le tableau 1 un résumé des principales caractéristiques de ces roches.

Par la variété de leur réseau poreux, les échantillons étudiés sont raisonnablement représentatifs des grands types de réservoirs carbonatés, à l'exception notable des dolomies et des craies.

\subsection{Dispositif expérimental}

Le dispositif expérimental, schématisé sur la figure 2, est conçu pour assurer deux fonctions principales :

- une mesure acoustique de grande précision relative, permettant de quantifier de très faibles variations de vitesses ; - une substitution de fluide aisée.

Nous avons utilisé la technique d'enrobage de l'échantillon dans le métal de Wood (alliage fusible à $70^{\circ} \mathrm{C}$ ) qui assure un couplage acoustique de très grande qualité entre l'échantillon et les transducteurs.

\subsubsection{Partie acoustique}

Des transducteurs doubles $\mathrm{P} / \mathrm{S}$, fixés pour toute la durée de l'expérience sur les faces externes de l'enrobage de métal de Wood, permettent d'enregistrer, pour chaque type de saturation, des signaux de bonne qualité (fig. 3). Pour chaque étape, on enregistre aussi l'écho du signal réfléchi à l'interface métal de Wood/roche afin de mesurer le temps de vol de l'onde dans le métal de Wood. Ceci permet de faire directement les corrections de «tête à tête » et met à l'abri de l'effet perturbateur d'une éventuelle variation des propriétés acoustiques du métal de Wood en cours d'expérience (vieillissement rapide toujours à craindre dans ce type d'alliage).

La mesure du module d'incompressibilité des liquides saturants est assurée par la mesure de la masse spécifique (pycnomètre) et de la vitesse de propagation $\left(V_{\mathrm{fl}}\right)$ de l'onde $P$ de l'effluent $\left(K_{\mathrm{fl}}=\rho V_{\mathrm{fl}}^{2}\right)$.

\subsubsection{Partie hydraulique}

L'écoulement des liquides pour substitution de fluide est réalisé perpendiculairement à la propagation d'onde et parallèlement à la direction de plus faible perméabilité en cas

TABLEAU 1

Caractéristiques des roches utilisées pour les expériences

Rocks used in the experiments

\begin{tabular}{|c|c|c|c|c|c|c|}
\hline Roche & $\mathbf{N}^{\circ}$ & Pétrographie & Type de porosité & $\phi(\%)$ & $\boldsymbol{K} \boldsymbol{H}^{*}$ & $K V^{*}$ \\
\hline Brauvilliers & $\mathrm{S} 1$ & Calcaire oolithique & $\begin{array}{l}\text { Typiquement trimodal: } \\
\text { Macroporosité interoolithe. } \\
\text { Abond. macro. intraool. (mold) } \\
\text { Micropor. intraoolithique }\end{array}$ & 41 & 15 & 2,2 \\
\hline Lavoux fin & $\mathrm{S} 3$ & Calcaire pelletoïdal & $\begin{array}{l}\text { Quasi unimodal: } \\
\text { Rare, fine macroporosité } \\
\text { Abond. micropor. intrapellets }\end{array}$ & 23 & 8 & 8 \\
\hline Saint-Pantaléon & S5 & $\begin{array}{l}\text { Calcaire bioclastique } \\
\text { grossier }\end{array}$ & $\begin{array}{l}\text { Abond. macropor. intergranul. } \\
\text { à fort rayon d'accès } \\
\text { Micropor. intrabioclaste }\end{array}$ & 35 & 4000 & 2200 \\
\hline Les Estaillades & S7 & $\begin{array}{l}\text { Calcaire bioclastique } \\
\text { moyen }\end{array}$ & $\begin{array}{l}\text { Typiquement bimodal: } \\
\text { Macropor. intergranulaire } \\
\text { Micropor. intrabioclaste }\end{array}$ & 30 & 250 & 250 \\
\hline Saint-Maximin & SM & $\begin{array}{l}\text { Calcaire bioclastique } \\
\text { à millioles }\end{array}$ & $\begin{array}{l}\text { Abondante macro. intergranul. } \\
\text { Fine macropor. intramillioles } \\
\text { Micropor. intrabioclaste }\end{array}$ & 40 & 1600 & 1600 \\
\hline Lavoux à grains & V3 & Calcaire oolithique & $\begin{array}{l}\text { Macropor. interoolithes. } \\
\text { Abondante micro. intraool. }\end{array}$ & 24 & 50 & 40 \\
\hline Andésite de Volvic & $\mathrm{R} 8$ & Andésite à texture fluidale & $\begin{array}{l}\text { Macropor. vacuoles dégazage } \\
\text { Micropor. très fine }\end{array}$ & 23 & 60 & 15 \\
\hline
\end{tabular}

* $K H$ perméabilité horizontale en $\mathrm{mD}, K V$ perméabilité verticale en $\mathrm{mD}$. 


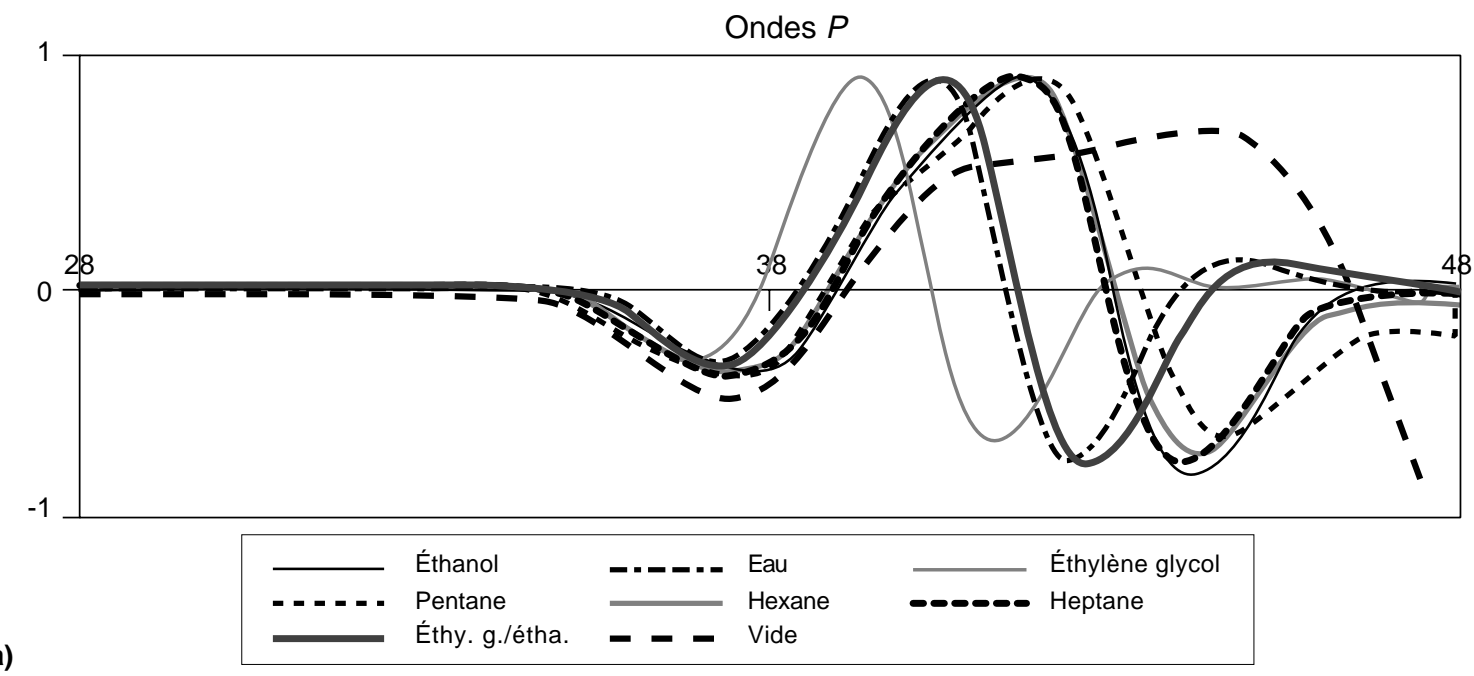

(a)

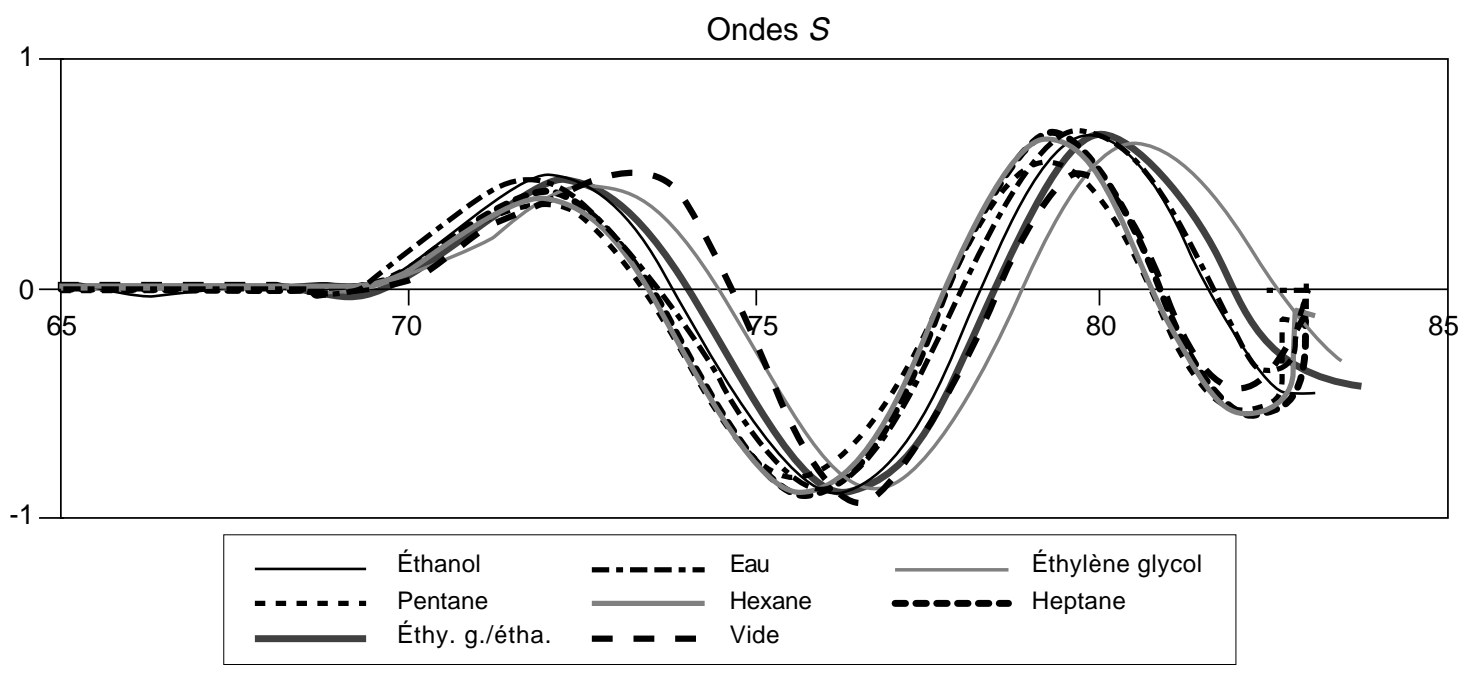

Figure 3

Exemple de signaux acoustiques (échantillon de calcaire de Saint-Maximin).

Example of recorded ultrasonic waves (Saint-Maximin limestone).

d'anisotropie. Il est facilité par la présence de plaque de verre fritté sur les faces d'accès du liquide.

L'échantillon enrobé, après avoir été mesuré (état « sec ») est évacué sous vide ( «état vide») puis totalement saturé d'eau. Les substitutions de liquide saturant s'effectuent de deux façons :

- par déplacement miscible, lorsque l'on veut obtenir un état de saturation monophasique. Cette situation correspond à la majorité des expériences présentées. À l'aide d'une pompe à débit constant ou d'un simple dispositif gravitaire, on assure l'écoulement d'un liquide miscible au précédent liquide saturant. On a utilisé diverses séries, comme: eau, éthylène glycol, mélanges d'éthanol et d'éthylène glycol en proportion variable pour faire varier
$K_{\mathrm{fl}}$, éthanol pur, liquides hydrocarbures légers (pentane, hexane, heptane), etc. Pour tous ces déplacements nous avons utilisé les critères suivants : déplacement d'au minimum 5 volumes de pores (VP) à des vitesses inférieures à 1,5 VP/j. Dans le cas du calcaire de Brauvilliers, qui présente des caractéristiques très originales au déplacement miscible (très longue traînée), nous avons triplé ces seuils de précaution, ce qui a conduit à une expérience de plus de quatre mois ;

- par déplacement forcé de l'eau non irréductible par une huile visqueuse, suivi de déplacements miscibles de liquides non miscibles à l'eau. Cette technique à été pratiquée sur l'échantillon S7 pour étudier l'état de saturation diphasique. 
Les caractéristiques des liquides utilisés sont données dans les figures correspondantes.

\subsubsection{Mesures des vitesses d'ondes pour le calcul des modules}

\section{Mesures de variations relatives de vitesse}

Pour un échantillon donné, on choisit un signal de référence correspondant à un état de saturation particulier dont on estime la vitesse absolue par pointé d'impétus. En corrélant les autres signaux par rapport à cette référence, on mesure les variations relatives de vitesse avec une très bonne précision. Lorsque la substitution de fluide n'entraîne pas de variation notable de l'atténuation du système (c'est-à-dire lorsque les deux signaux ont des spectres fréquentiels très proches), la corrélation permet d'estimer des variations de temps de vol avec une précision nettement meilleure que $100 \mathrm{~ns}$.

Mais il est fréquent que l'atténuation globale, surtout pour les ondes $P$, varie avec le fluide saturant (voir figure 3, pour laquelle nous avons choisi un exemple de variations particulièrement marquées). La modification de la forme du signal peut devenir suffisamment importante pour perturber la corrélation. Dans ce cas, la corrélation est centrée sur la première demi-période du signal, voire sur le premier quart de période. Il existe une technique permettant de s'affranchir, au moins théoriquement, de cette difficulté. Il s'agit de la méthode de mesure de vitesse de phase, fondée sur la différence de phase mesurée par rapport de spectre entre le signal de référence et le signal analysé. Nous n'avons pas utilisé cette technique, à ce stade de l'étude, pour des raisons pratiques mais aussi parce que les incertitudes sur les variations de vitesse dues aux substitutions de fluides restent faibles.

Dans le cas de signaux assez différents de la référence, l'incertitude est de l'ordre de $100 \mathrm{~ns}$. En y ajoutant une incertitude de 100 ns liée à l'imprécision des pointés d'échos, on peut estimer à $200 \mathrm{~ns}$ l'incertitude globale maximale pour des temps de vol de l'ordre de 12 à $15 \mathrm{~ms}$ (onde $P$ ) et 21 à $26 \mathrm{~ms}$ (ondes $S$ ) conduisant à des incertitudes relatives de l'ordre de $1,5 \%$ sur $P$ et $1 \%$ sur $S$. La précision de mesure des masses volumiques étant très grande, l'incertitude sur le module de cisaillement $\mu$ où $V s$ intervient au carré sera donc de l'ordre de $2 \%$. Concernant l'incompressibilité, pour laquelle il faut cumuler les incertitudes $P$ et $S$, on atteint $5 \%$. Cela nous conduit sur $K_{\text {sat }}$ à des barres d'erreurs inférieures à $1 \mathrm{GPa}$ dans le pire des cas, alors que l'amplitude de variation attendue dépasse les $5 \mathrm{GPa}$. Comme nous nous attachons à décrire une droite de régression expérimentale dont chaque point est indépendant de ses voisins en ce qui concerne les incertitudes, cette imprécision ne devrait en aucun cas occulter le phénomène recherché.

\section{Le problème de la détermination absolue des vitesses}

La conclusion optimiste précédente n'est bien sûr valable qu'en ce qui concerne les variations relatives des vitesses par rapport à la valeur absolue de vitesse de l'échantillon de référence. La mesure de la vitesse absolue, en laboratoire, est un problème souvent sous-estimé. Si l'on procède, comme classiquement, par pointé d'impétus, les incertitudes expérimentales sont très faibles. Mais la signification de cette mesure est douteuse puisque l'effet bien connu de «dispersion de cheminement» (voir par exemple Cadoret et $a l ., 1995)$ peut conduire à des impétus trop précoces. Tel est le cas si une très faible partie de l'énergie acoustique emprunte un chemin plus rapide, lié à une hétérogénéité de la roche de dimension caractéristique non négligeable devant la longueur d'onde. L'incertitude sur la vitesse absolue, causée par la dispersion de cheminement, peut dépasser $5 \%$, notamment dans les roches calcaires. Nous devons nous interroger sur les conséquences de cette incertitude sur notre expérience.

\section{Approche par calcul}

Prenons le cas d'un échantillon vérifiant la loi de Gassmann linéarisée $K_{\mathrm{sat}}=K_{\mathrm{dry}}+a K_{\mathrm{fl}}$.

Les variations relatives de vitesse (expérimentalement : différences de temps de vol) pour les différents $K_{\mathrm{fl}}$ sont parfaitement connues par rapport à une référence dont les vitesses absolues $P$ sont entachées d'une erreur de $\mathrm{n} \%$. Il est très facile de tracer le graphe de la figure 4 , pour lequel nous avons choisi les caractéristiques standard en modules et porosité $\left(K_{\text {dry }}=8 \mathrm{GPa}, \mu=6 \mathrm{GPa}, \phi=0,3\right)$. Nous avons calculé les conséquences d'une erreur de + et $-5 \%$ sur la mesure de la vitesse absolue de trois signaux de référence (choisis pour des $K_{\mathrm{fl}}$ de $1,2,3 \mathrm{GPa}$ ).

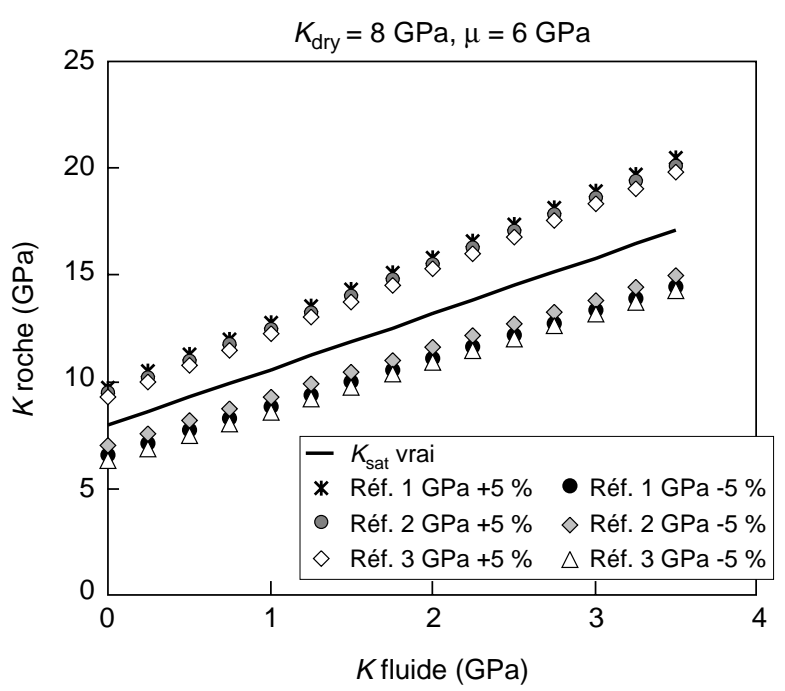

Figure 4

Exemple d'erreur induite par l'incertitude sur la vitesse absolue de la référence.

Example of error induced by the uncertainty on the reference velocity absolute value. 
On constate que les conséquences sont très peu sensibles à la référence choisie (on aurait pu penser que l'effet varierait suivant que la référence était « rapide» ou «lente»). Les effets sur les trois paramètres $K_{\text {dry }}, \beta$, et $K_{\text {grain }}$ dérivés sont quantitativement très contrastés (tableau 2 ).

\section{TABLEAU 2}

Effets de l'incertitude de valeur absolue de vitesse sur le calcul des paramètres pétroacoustiques

Impact of the reference velocity absolute value uncertainty on the petroacoustic parameters computation

\begin{tabular}{c|c|c}
\cline { 2 - 3 } & \multicolumn{2}{c}{$\begin{array}{c}\text { Variation de la vitesse absolue } \\
\text { de la référence }\end{array}$} \\
\cline { 2 - 3 } & $\mathbf{+ 5 \%}$ & $\mathbf{- 5 \%}$ \\
\hline Variation $K_{\text {dry }}$ & $20 \%$ & $-16 \%$ \\
\hline Variation $\beta$ & $8 \%$ & $-7 \%$ \\
\hline Variation $K_{\text {grain }}$ & $188 \%$ & $-35 \%$ \\
\hline
\end{tabular}

Nous en tirerons deux conclusions en ce qui concerne notre vérification expérimentale :

- Les conséquences sur $K_{\text {dry }}$ sont logiquement celles que prévoit un simple calcul d'erreur sur la formule $K=\rho V p^{2}$ $-(4 / 3) \mu$.

- Les conséquences sur $K_{\text {grain }}$ sont « catastrophiques ». On ne pourra donc pas utiliser strictement la valeur calculée de $K_{\text {grain }}$ pour valider nos résultats expérimentaux. $A$ contrario, même si la valeur de $K_{\text {grain }}$ tirée de l'expérience s'éloigne de la «norme», cela ne constituera pas un fort argument d'échec.

\section{Approche expérimentale}

Nous pouvons, partiellement, réaliser une estimation expérimentale des effets de l'incertitude sur la vitesse absolue des références, en utilisant successivement chaque état de saturation comme référence de corrélation. La figure 5 montre le résultat pour l'échantillon de calcaire de Saint-Maximin, dont les signaux sont présentés sur la figure 3. Les vitesses absolues des références ont été calculées à partir du pointé d'impétus «brut» mesuré en cours d'expérience. On peut observer que :

- pour les modules de cisaillement, les résultats sont remarquablement homogènes, l'écart maximum entre les diverses références est de 0,4 GPa. Ces constatations nous montrent seulement que le pointé d'impétus d'onde $S$ est très peu dépendant de l'état de saturation, ce qui est normal puisque le module de cisaillement est très peu sensible au liquide saturant. En revanche, cela ne nous apprend rien sur un éventuel effet de dispersion de cheminement ;

- pour les modules d'incompressibilité, l'écart maximum est de l'ordre de 1,5 GPa;

- si l'on considère les résultats déduits des droites de régression (fig. 5), en excluant les valeurs à l'état de «saturation» vide, l'on observe des résultats cohérents avec nos conclusions du paragraphe précédent. La variation sur $K_{\text {dry }}$ est faible (mais nous n'avons pas de référence absolue). La valeur calculée pour $K_{\text {grain }}$ varie du simple au double, mais en restant centrée autour de la norme pour la calcite (70 GPa). Il suffirait de faire varier de 1 ou $2 \%$ le temps de vol dans certaines références, pour trouver une valeur proche de $70 \mathrm{GPa}$. Cette variation de 1 ou $2 \%$ sur le temps absolu n'est pas choquante, si l'on considère que l'on peut ajouter les incertitudes liées au temps de vol dans le métal

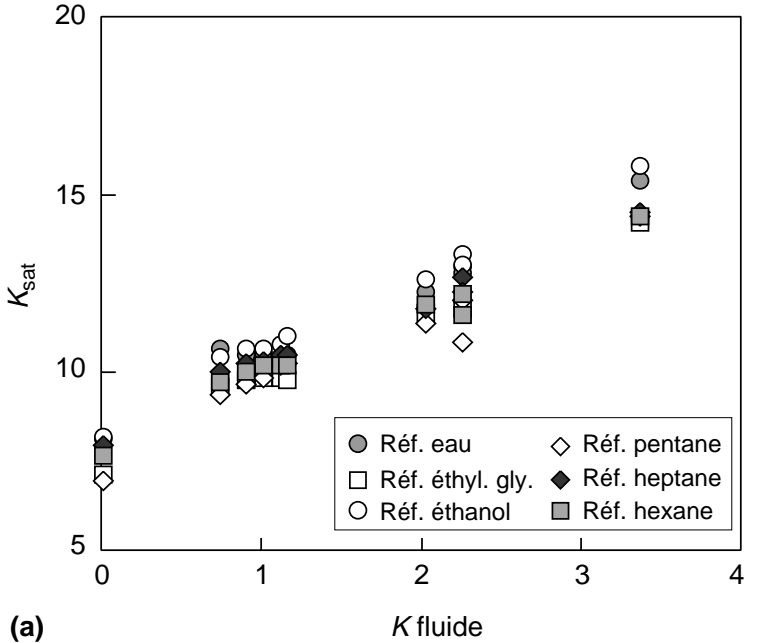

Figure 5

Exemple de corrélation sur références multiples (Saint-Maximin). Example of correlation on multiple references (Saint-Maximin limestone).

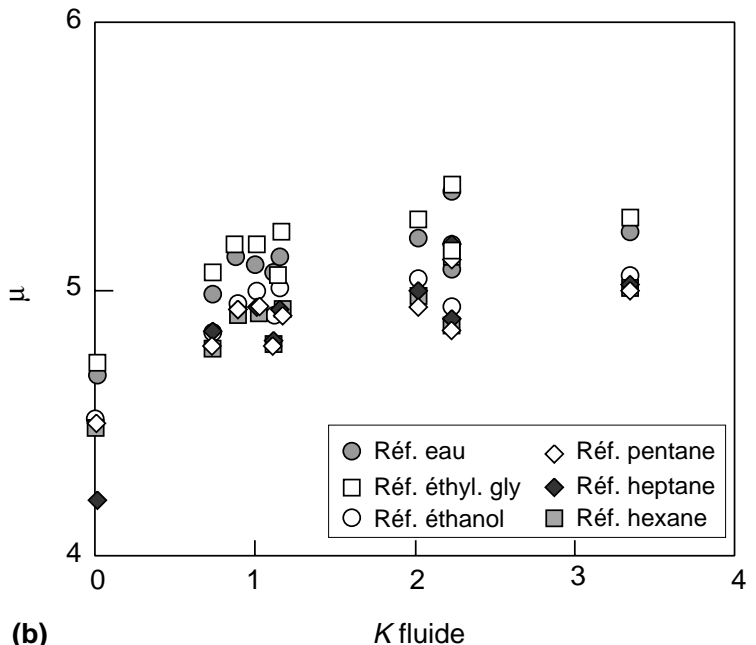

(b)

$K$ fluide 
de Wood (estimé par la méthode de l'écho) et les éventuels effets de dispersion de cheminement dans la roche.

\section{RÉSULTATS SUR SIX CALCAIRES ET UNE LAVE POREUSE}

\subsection{Mode de représentation des résultats}

Compte tenu des remarques précédentes, nous avons adopté, pour les résultats expérimentaux, la méthode de représentation suivante. Après avoir réalisé les corrélations multiples, comme présenté sur la figure 5, nous avons sélectionné les quatre références donnant les résultats les mieux groupés. C'est la moyenne de ces résultats que nous présentons sur les figures 6 et 7 .

En observant les résultats, on constate que deux états de saturation posent des problèmes particuliers :

- L'état de saturation sec atmosphérique ou sec vide. Cela n'est pas étonnant puisque nous avons déjà signalé que, dans cet état, les forces capillaires associées à l'eau adsorbée jouaient un rôle important, non pris en compte par la formule de Gassmann. Le cas de l'andésite est frappant puisque le module d'incompressibilité varie presque du
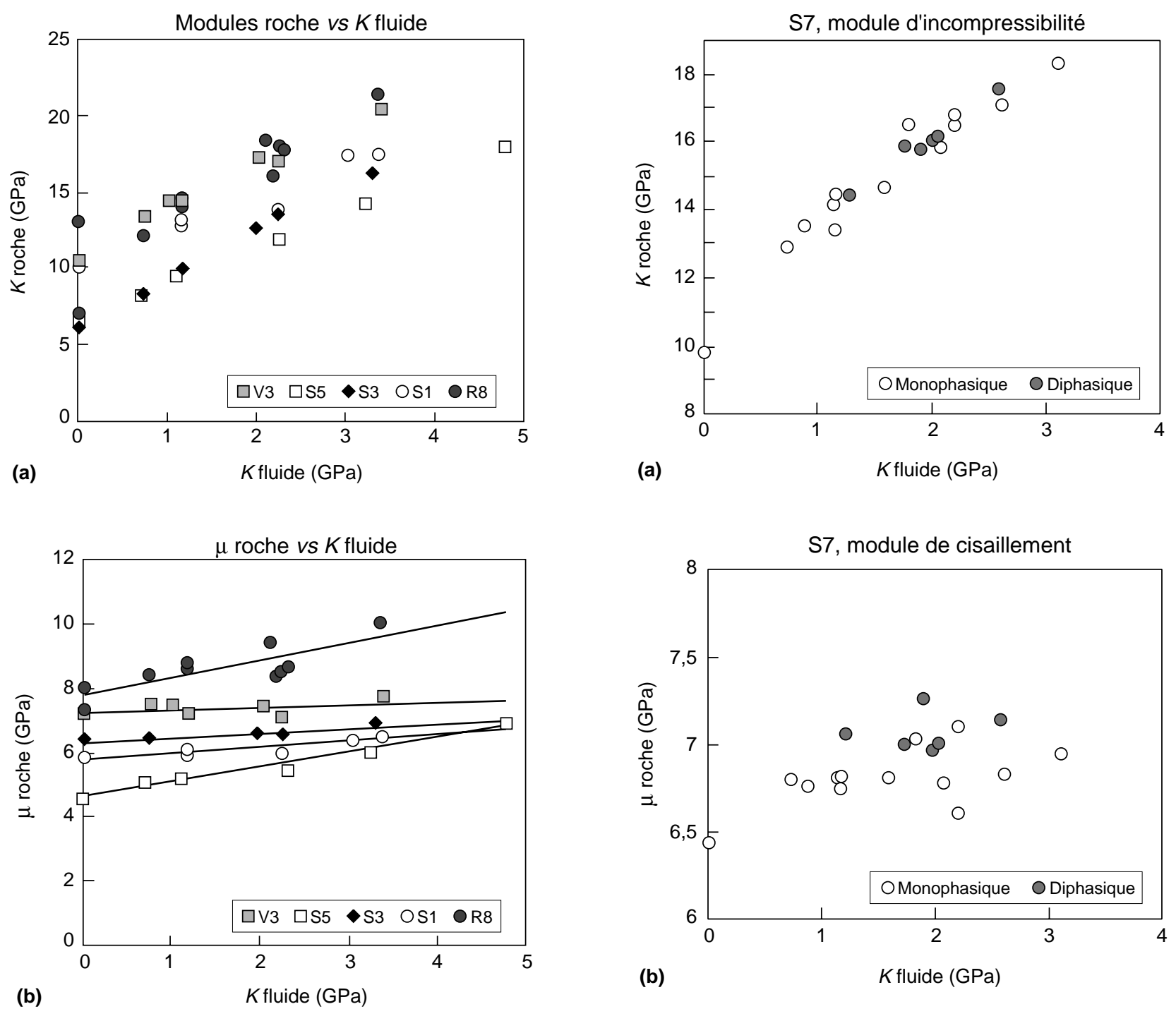

Figure 6

Relations expérimentales modules $=f\left(K_{\mathrm{fl}}\right)$ pour quatre calcaires et une andésite.

Bulk and shear modulus vs. $K_{f l}$ relationships in four limestone sample and one porous lava.

Figure 7

Relations expérimentales modules $=f\left(K_{\mathrm{fl}}\right)$ pour le calcaire des Estaillades, saturations monophasiques et diphasiques. Bulk and shear modulus vs. $K_{f l}$ relationships in Les Estaillades limestone. Monophasic and diphasic saturation. 
simple au double entre l'état sec atmosphérique et l'état sec vide. Remarquons toutefois qu'en ce qui concerne les calcaires, les variations sont infiniment plus faibles et que le module d'incompressibilité à l'état sec s'intègre assez bien au reste de la courbe. Ceci est dû au fait que les microdéfauts, à l'origine de cette dépendance des propriétés mécaniques par rapport à la capillarité, sont plutôt rares dans les roches calcaires.

- L'état de saturation totale en eau. Nous avons constaté expérimentalement une variabilité anormale du résultat à l'état de saturation en eau. Une première cause est à rechercher dans le fait que, l'expérience commençant par la saturation en eau, des traces d'air peuvent persister, au début, dans l'échantillon, faisant que le $K_{\mathrm{fl}}$ réel est inférieur au $K_{\mathrm{fl}}$ mesuré sur l'eau de saturation. Cette explication est satisfaisante pour les cas où une nouvelle saturation, bien postérieure, permet de mesurer des incompressibilités «normales ». Mais dans certain cas, l'incompressibilité mesurée pour l'état de saturation en eau, lors du retour à cette saturation en cours d'expérience, parait légèrement inférieure à la tendance générale. Cette observation (qui reste quantitativement peu importante) pose le problème de la nature réelle des molécules adsorbées sur les microdéfauts mécaniques lorsque la roche est saturée de liquide fortement miscible à l'eau (éthanol, par exemple). D'un point de vue pratique, par précaution, nous exclurons les incompressibilités mesurées à l'état saturé en eau des calculs de droite de régression des points expérimentaux. Bien évidemment, et pour des raisons plus sérieuses, les valeurs d'incompressibilité à l'état sec ou vide seront également exclues de ces régressions.

\subsection{Résultats généraux}

\subsubsection{Concernant les modules de cisaillement}

Les résultats sont reportés sur les figures 6 et 7. On constate, pour les échantillons S1, S3, S7, SM et V3, une claire indépendance de $\mu$ par rapport au fluide saturant. On vérifie donc la règle classique. Deux échantillons semblent toutefois $\mathrm{y}$ faire exception. Il s'agit du calcaire de Saint-Pantaléon (S5) et de l'andésite de Volvic (R8). Mais le cas du S5 ne pose pas de vrai problème, puisque l'effet de fluide sur $\mu$, est essentiellement lié à l'utilisation de glycérol dont la viscosité est suffisamment importante $(1500 \mathrm{cP})$ pour influencer les ondes $S$ de fréquence ultrasonore.

Le cas de l'andésite de Volvic (R8) est plus intéressant. La dérive d'environ $2 \mathrm{GPa}$ sur la valeur de $\mu$, entre la saturation en air et en éthylène glycol, est trop importante pour être attribuée à une dérive de mesure. La relation entre l'incompressibilité et la viscosité est suffisamment grande pour que l'on puisse lire la figure 6 comme un graphique: module de torsion en fonction de la viscosité. Bien que la viscosité maximale utilisée dans l'expérience, celle de l'éthylène glycol, soit relativement faible $(19 \mathrm{cP})$, on peut observer, dans l'andésite de Volvic, un effet de viscosité sur le module de cisaillement. Cela est très certainement lié à la présence de microdéfauts d'un genre particulier.

\subsubsection{Concernant les modules d'incompressibilité (effet G assmann stricto sensu)}

L'observation la plus importante que l'on peut faire sur les figures 6 et 7, est, bien sûr, la linéarité de la relation $K_{\text {sat }}$ $=f\left(K_{\mathrm{fl}}\right)$. Nous avons là une première vérification qualitative de la formule de Gassmann dans sa version simplifiée. Disons plus précisément que l'on vérifie une condition nécessaire (mais non suffisante).

Pour aller plus avant, il faut considérer les résultats quantitatifs dérivés des droites $K_{\mathrm{sat}}=f\left(K_{\mathrm{fl}}\right)$, qui sont résumés dans le tableau 3 .

Le seul paramètre pour lequel nous avons a priori une information quantitative, est le module d'incompressibilité de grain $\left(K_{\text {grain }}\right)$. Il est d'environ $70 \mathrm{GPa}$ pour la calcite constituant nos six calcaires. Notons toutefois que les valeurs compilées dans la littérature par Mavko et al. (1998) varient entre 63,7 et 76,8 . Nous ne disposons d'aucune information concernant l'andésite.

Nous avons montré l'extrême sensibilité de ce paramètre aux incertitudes sur la valeur absolue de vitesse de référence

TABLEAU 3

Résultats quantitatifs dérivés des régressions expérimentales $K_{\mathrm{sat}}=f\left(K_{\mathrm{fl}}\right)$

Results derived from experimental $K_{\text {sat }}=f\left(K_{f l}\right)$ relationships

\begin{tabular}{|c|c|c|c|c|c|c|c|}
\hline & $\begin{array}{c}\text { Lavoux à grains } \\
\text { V3 }\end{array}$ & $\begin{array}{l}\text { Lavoux fin } \\
\text { S3 }\end{array}$ & $\begin{array}{c}\text { Saint-Maximin } \\
\text { SM }\end{array}$ & $\begin{array}{c}\text { Saint-Pantaléon } \\
\text { S5 }\end{array}$ & $\begin{array}{l}\text { Les Estaillades } \\
\text { S7 }\end{array}$ & $\begin{array}{c}\text { Brauvilliers } \\
\text { S1 }\end{array}$ & $\begin{array}{c}\text { Andésite de Volvic } \\
\text { R8 }\end{array}$ \\
\hline Coeff. cor. & 1 & 1 & 1 & 1 & 0,97 & 0,99 & 0,98 \\
\hline Pente & 2,68 & 3,12 & 1,86 & 2,34 & 2,25 & 2,14 & 3,3 \\
\hline Porosité & 0,23 & 0,24 & 0,4 & 0,34 & 0,3 & 0,41 & 0,22 \\
\hline$K_{\text {dry }}(\mathrm{GPa})$ & 11,30 & 6,24 & 8,35 & 6,8 & 11,55 & 10,51 & 10,31 \\
\hline$\beta$ & 0,8 & 0,87 & 0,86 & 0,89 & 0,82 & 0,93 & 0,84 \\
\hline$K_{\text {grain }}$ & 56 & 47 & 62 & 63 & 65 & 160 & 64 \\
\hline
\end{tabular}


(Section Approche par calcul). Aussi, peut-on considérer comme particulièrement satisfaisantes les valeurs de $K_{\text {grain }}$ dérivées de la courbe expérimentale. Seule la valeur correspondant au calcaire de Brauvilliers (S1) s'écarte de l'ordre de grandeur prévu, mais, comme nous l'exposions précédemment pour le calcaire de Saint-Pantaléon (S5), une variation de quelques pour-cent sur les valeurs absolues de vitesses de référence justifie largement cet écart.

La linéarité de la relation $K_{\mathrm{sat}}=f\left(K_{\mathrm{fl}}\right)$ et la bonne concordance quantitative de la valeur de $K_{\text {grain }}$ dérivées de la pente expérimentale avec celles données pour la calcite, dans la littérature, constituent une vérification expérimentale indiscutable de la formule de Gassmann.

On peut noter, sur le tableau 3, l'absence de relation apparente entre le module $K_{\text {dry }}$ et la porosité. Nous voyons là le reflet amplifié (puisqu'un module est proportionnel au carré d'une vitesse) de la faible dépendance des vitesses par rapport à la porosité observée dans le cas des calcaires poreux et parfois exprimé sous la rubrique «porosité secondaire» dans la formule classique de Wyllie, reliant porosité et vitesse mesurée sur diagraphie sonique. Il s'agit d'un sujet d'un grand intérêt pour les applications géophysiques, mais qui dépasse de beaucoup le cadre de cet exposé.

\subsection{Cas des saturations diphasiques}

Sur l'échantillon de calcaire des Estaillades (S7), nous avons réalisé des mises en saturation diphasiques, par déplacement forcé d'une huile paraffinique visqueuse (200 cP environ). En prenant pour valeur de $K_{\mathrm{fl}}$ la moyenne harmonique des modules d'incompressibilité des liquides, pondérée par les saturations correspondantes, les points expérimentaux s'alignent remarquablement avec les points correspondant aux saturations monophasiques (fig. 7). Nous avons là une preuve de la validité de la formule de Gassmann, même dans un cas de saturation diphasique (deux fluides non miscibles). Ce point est important, puisque cette condition de saturation (qui ne vérifie pas exactement les hypothèses de base de calcul de Gassmann) correspond au cas le plus général des réservoirs de pétrole ou de gaz.

\section{CONCLUSION}

Nous voudrions insister sur deux points qui nous paraissent constituer les principaux résultats de cette étude.
- Tout d'abord, ces expériences réalisées sur un ensemble de sept types de roches (six échantillons de calcaires et un échantillon de lave) nous apportent une vérification expérimentale très convaincante de la formule de Gassmann. Cette vérification étant réalisée aux fréquences ultrasonores, et le calcul de Gassmann correspondant à des conditions quasi statiques, on peut donc très raisonnablement en déduire la validité pour les fréquences sismiques.

- Mais aussi, la méthode présentée peut constituer une très bonne méthode de mesure des paramètres poroélastiques des échantillons de carotte. La principale modification à apporter est la mise en pression de confinement, qui ne pose pas de réels problèmes. L'utilisation de cette méthode nous a fourni d'excellents résultats.

\section{REMERCIEMEN TS}

Ce travail a été réalisé au laboratoire de Physique des roches de l'Institut français du pétrole, avec l'aide de Michel Masson que nous remercions chaleureusement. Une partie de ces travaux a bénéficié d'une aide financière européenne dans le cadre du projet SACS2 (Séquestration géologique du $\mathrm{CO}_{2}$ ).

\section{RÉFÉREN CES}

Biot, M.A. (1956). Theory of Propagation of Elastic Waves in Afluid-Saturated Porous Solid. J. Acoustical Soc. Am., 28, 168191.

Bourbié, T., Coussy, O., Zinszner, B. (1986) Acoustique des milieux poreux, Éditions Technip.

Cadoret, T. (1993) Effet de la saturation eau/gaz sur les propriétés acoustiques des roches. Thèse, Paris VII.

Cadoret, T., Marion, D. and Zinszner, B. (1995) Influence of Frequency and Fluid Distribution on Elastic Wave Velocities in Partially Saturated Limestones. J. Geophys. Res., 100, B6.

Gassmann, F. (1951) Uber die Elastizitatporosen Medien. Vierteljahrsschrift der Naturforschenden Gesellshaft in Zurich, 96, 1-23.

Lucet, N. (1989) Vitesse et atténuation des ondes élastiques soniques et ultrasoniques dans les roches sous pression de confinement. Thèse, Paris VI.

Mavko, G., Mukerji, T., Dvorkin, J. (1998) Rock Physics Handbook, Cambridge University Press.

Tittmann, B., Clark, V., Richardson, J., and Spencer, T. (1980). Possible Mechanism for Seismic Attenuation in Rocks Containing a Small Amount of Volatiles. J. Geophys. Res., 85, 5199-5208.

Manuscrit définitif reçu en février 2002 